\title{
Corrigendum
}

\section{lodised salt contribution to iodine nutrition status of pregnant and lactating women - CORRIGENDUM}

Haihong Zhang, Shengmin Lv, Zhenguo Mu, Weihong Li, Xia Zhang, Yuchun Wang and Shannon Rutherford

(First published online 11 January 2016)

doi:10.1017/S0007114515001543, Published by Cambridge University Press, 12 September, 2015.

As Haihong Zhang is the principal researcher of this study, we would like to assign her as the corresponding author. Shengmin Lv is no longer the corresponding author.

Corresponding author: HH. Zhang, Shijiazhuang Municipal Center for Disease Prevention and Control, No. 3, Likang Street, Shijiazhuang City, 050011, Hebei Province, People's Republic of China. Fax: 8631186035727. Email: dbk8419@263.net

The authors apologise for this error.

\section{Reference}

Zhang H, Lv S, Mu Z, et al. (2015) Iodised salt contribution to iodine nutrition status of pregnant and lactating women. British Journal of Nutrition 114, 126-133. doi:10.1017/S0007114515001543. 